\title{
Strongly coupled dusty plasmas: finite and extended systems
}

\author{
A. Melzer*, S. Käding ${ }^{*}$, D. Block ${ }^{\dagger}$, A. Piel ${ }^{\dagger}$, P. Ludwig** and M. Bonitz** \\ *Institute of Physics, University Greifswald, 17487 Greifswald, Germany \\ ${ }^{\dagger}$ IEAP, Christian-Albrechts-Universität, 24098 Kiel, Germany \\ ${ }^{* *}$ ITAP, Christian-Albrechts-Universität, 24098 Kiel, Germany
}

\begin{abstract}
Systems of confined microspheres in a gaseous plasma environment are studied. These systems of trapped "dust" particles can form extended structures where the size of the system is much larger than interparticle distance. With specially tailored confinement potentials also finite arrangements of microspheres can be formed. In these systems the structure and dynamics dramatically depend on the exact particle number. Here, three-dimensional finite systems of microspheres, so-called Yukawa balls, are investigated and their structural properties are discussed with respect to the realized configurations of ground and metastable states.
\end{abstract}

Keywords: dusty plasma, dust cluster, normal modes

PACS: $52.27 . L w, 36.40 .-\mathrm{c}$

\section{INTRODUCTION}

Microscopic particles ("dust") immersed in a gaseous plasma environment are found in a wide variety of systems. Dusty plasmas are ubiquitous in astrophysical situations, like planetary rings, comets, interstellar clouds [1]. Dust in plasmas plays also a crucial role in industrial processes such as thin film deposition or plasma etching [2]. There, typically, the removal of the dust particles from the process chamber is a critical issue. Finally, dusty plasmas provide an ideal tool to study fundamental properties of condensed matter, like crystallization properties, plasma-induced forces or particle dynamics [3]. In dusty plasmas a number of novel dust and plasma phenomena are observable [4].

The dust particles in the plasma attain high negative charges due to the continuous inflow of electron and ions. Typical charges are of the order of 10000 elementary charges for a 10 micron dust particle. Ordered states of trapped charged particles are formed when the coupling parameter $\Gamma=Z^{2} e^{2} / 4 \pi \varepsilon_{0} b k T$ exceeds a critical value, $\Gamma_{\text {crit }} \simeq 180$ for infinite systems. Here, $Z$ is the charge number of the particles and $b$ the interparticle spacing. The coupling parameter is the electrostatic energy of neighboring particles in units of the thermal energy $k T$. Due to their high charge the dust particles can crystallize into ordered arrangements even at room temperature forming so-called plasma crystals $[5,6,7]$.

The spatial and time scales of the particle motion allow easy observation by video microscopy. Weak frictional damping ensures that the dynamics and kinetics of individual particles become observable. Thus, dusty plasmas enable the investigation of crystal structure, solid and liquid plasmas, phase transitions, waves and many more phenomena on the kinetic particle level.

\section{EXTENDED AND FINITE SYSTEMS}

In the plasma, the particles are subject to a number of forces. For the micrometer sized particles typically used in studies on fundamental properties of dusty plasmas gravity and electric field forces are dominant. Another relevant force in the plasma bulk is the ion drag force which is a force in the direction of the ion flow due to Coulomb and direct collisions between streaming ions and the dust. Finally, also the thermophoresis due to temperature gradients in the neutral gas background that drives the particles towards colder regions is often applied in dusty plasmas. By tailoring all these forces, finite and extended dust structures can be generated.

Extended flat (2D) dust crystals are formed in the space charge sheath above the flat electrode of parallel plate RF discharges $[8,6,7,9]$. In these discharges, usually the sheath electric field is strong enough to balance the weight of the particles. Since the mean electric field in the sheath generally increases towards the electrode a force balance is achieved at a unique vertical position in the sheath. Due to the strong vertical forces the dust is confined to a flat structure. 
a)

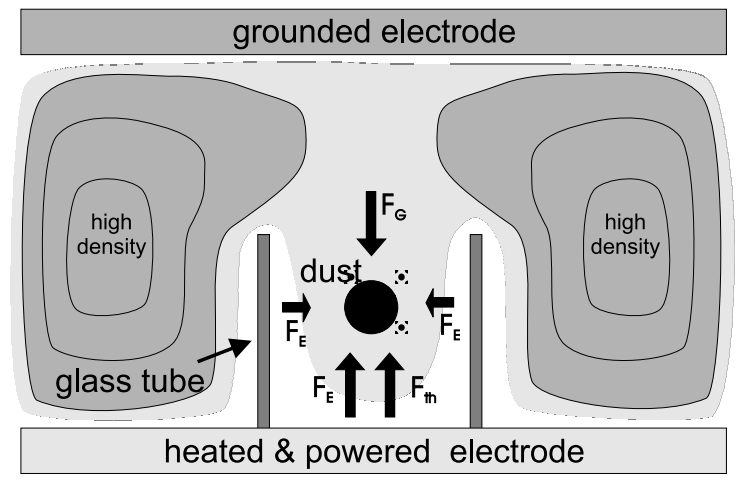

b)

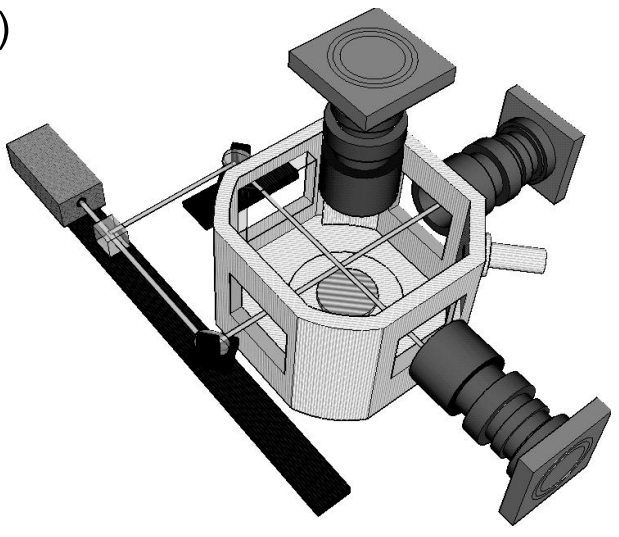

FIGURE 1. Scheme of the confinement of Yukawa balls [36]. a) A glass cube is placed on the lower electrode of an rf discharge. The lower electrode is heated to compensate gravity. The combined action of gravity, electric forces and thermal gradients provide an isotropic confinement for the microspheres. b) The microspheres are illuminated by an expanded laser beam and the particle motion is recorded by three orthogonal high-speed cameras.

These $2 \mathrm{D}$ systems preferably arrange in a hexagonal structure $[6,8,7,9,10]$ which is the ground state configuration of $2 \mathrm{D}$ crystals. In multilayer systems also other crystal structures like fcc or bcc have been identified [8, 11, 12]. Moreover, vertically aligned structures due to the directed ion streaming motion in the sheath are frequently observed $[10,13]$. Phase transitions $[14,15]$ and various types of waves have been studied, see e.g. [4] and references therein.

Consequently, extended three-dimensional (3D) dust structures are found when dusty plasmas are investigated under microgravity conditions aboard parabolic flights [16, 17, 18, 19] or on the International Space Station [20, 21, 22, 23, 24]. There, the particles are confined to the plasma bulk by the much weaker ambipolar electric field. However, the central plasma region is found to be dust-free. The dust is expelled from this so-called "void" and a sharp boundary between the void and the dust cloud is formed (see e.g. [16]). The void boundary is generally considered to be due to the force balance of the inward electric field force and the outward ion drag force [25, 26, 27, 28]. In the 3D dust cloud, for example, the structure [29], charging processes [30,31] and self-excited waves have been investigated [22, 18].

Finite dust systems (dust clusters) are obtained when only a small number of particles are trapped in bounded confinement potentials. Clusters have been generated in two dimensions (2D) e.g. by Refs. [32, 33, 34, 35]. There, as an example, a shallow circular parabolic depression has been manufactured into the electrode. This depression distorts the equipotential lines in the sheath and provides a radial (horizontal) confinement of the dust. These 2D finite systems have been studied e.g. with respect to their structure, rotational stability and normal modes $[32,33,34,35]$.

Similarly, linear (1D) systems have been generated by placing a narrow rectangular barrier on the electrode [37, 38]

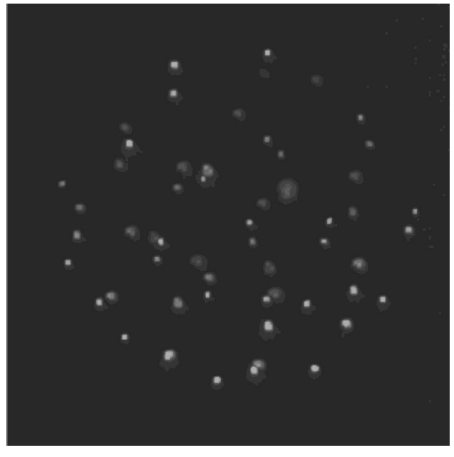

front

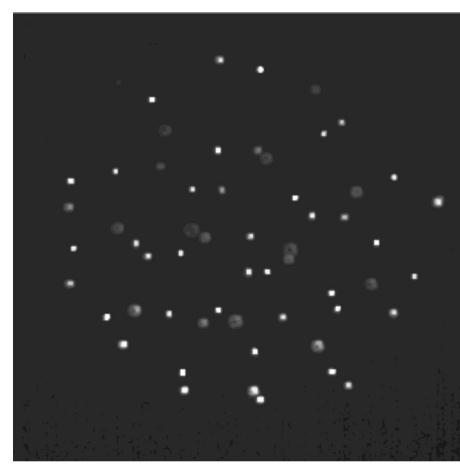

side

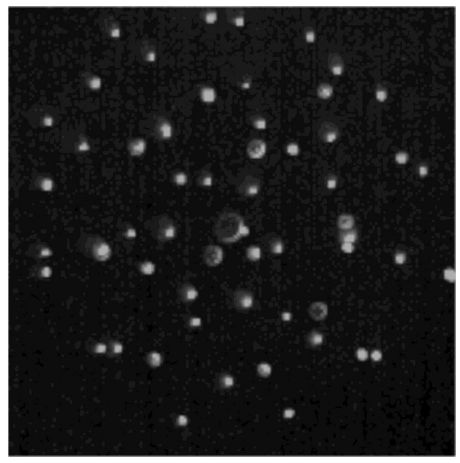

top

FIGURE 2. Snap shots of a Yukawa ball with about $N=60$ particles. From the images of the front, side and top view camera the 3D particle positions are calculated. 
or by a groove in the electrode, see e.g. [39, 40]. Wave propagation, structural transitions and the phonon spectrum have been studied in these systems.

Recently, ball-shaped 3D clusters (so-called Yukawa balls) have been successfully generated in the laboratory $[36,41]$. These Yukawa balls are not hampered by a dust-free void in their center. Rather they form a compact convex structure with concentric "onion" shells [36]. These very interesting systems will be described in more detail below.

\section{EXPERIMENT}

The experiments on Yukawa balls have been conducted in a parallel plate capacitively coupled rf-discharge at $13.56 \mathrm{MHz}$ in argon at a gas pressure of $p=90 \mathrm{~Pa}$ and a discharge power of $P=7.5 \mathrm{~W}$. Monodisperse plastic microspheres of $3.46 \mu \mathrm{m}$ diameter are dropped into the discharge.

A complex interplay of forces is used to confine the dust particles in a Yukawa ball [42]. The horizontal confinement is achieved by a cubic glass tube placed on the lower electrode (see Fig. 1a). The electric forces due to the dielectric glass walls provide a radial inward force on the dust. Vertically, gravity is compensated by the combined action of an upward thermophoretic force and a weaker electric field force. The thermophoretic force is generated by heating the lower electrode to temperatures of about $50-70^{\circ} \mathrm{C}$. The vertical electric field is due to the sheath formation above the lower electrode. The combined horizontal and vertical forces form a 3D isotropic harmonic potential well for the dust particles [42]. In this trap, the microspheres form Yukawa balls with nested concentric spherical shells.

To record images of the Yukawa balls, a novel stereoscopic camera setup is used that consists of three synchronized cameras with pairwise perpendicular orientation (see Fig. 1b). The cameras have megapixel resolution and allow a frame rate up to 500 frames per second. The dynamical processes in Yukawa balls are very slow so that a frame rate of up to 10 frames per second was sufficient. The whole particle cloud is illuminated by an expanded circular laser beam $(600 \mathrm{~mW}$ at $532 \mathrm{~nm})$. With this setup the 3D positions of all particles in a Yukawa ball can be measured simultaneously with high spatial and temporal resolution [43, 44]. Presently, Yukawa balls with up to $N=100$ microspheres can be analyzed. In previous experiments, a scanning technique has been used where successive slices of the Yukawa ball have been recorded and analyzed. This scanning technique is non-simultaneous, but it allows to measure the configuration of crystalline Yukawa balls where the particle motion is slow.

Figure 2 shows snap shots of the Yukawa ball recorded by the front, side and top view camera. In a first step the particle positions in each of the camera images are determined. Combining the information from the three orthogonal cameras the 3D particle positions are calculated.

\section{STRUCTURE AND OCCUPATION NUMBERS}

Examples of Yukawa balls are shown in Fig. 3. There, Yukawa balls with $N=31,52$ and 91 particles are shown. It is seen, that the Yukawa balls indeed arrange in concentric shells (see also [36]). The $N=31$ cluster shown here has a configuration $(5,26)$ where 5 particles are on the inner shell and 26 on the outer. The $N=52$ cluster has three shells with a configuration $(1,12,39)$. Finally, the $N=91$ cluster has a three-shell configuration with $(4,27,60)$.

It is interesting to compare the observed structures with simulations [45, 46, 47, 48]. From the structure, especially from the occupation number of the different shells, the interaction strength can be deduced [48].

Yukawa balls are usually modeled from the energy of the system

$$
E=\frac{1}{2} m \omega_{0}^{2} \sum_{i=1}^{N} r_{i}^{2}+\frac{Z^{2} e^{2}}{4 \pi \varepsilon_{0}} \sum_{i>j}^{N} \frac{\exp \left(-r_{i j} / \lambda_{\mathrm{D}}\right)}{r_{i j}}
$$

Here, $m=3.28 \times 10^{-14} \mathrm{~kg}$ is the mass of the dust particles and $\omega_{0}$ is the resonance frequency of the confinement potential for the dust. Further, $r_{i}=\left|\vec{r}_{i}\right|$ is the radial position of the $i$ th particle and $r_{i j}=\left|\vec{r}_{i}-\vec{r}_{j}\right|$ is distance between particles $i$ and $j$. The first term on the RHS describes the potential energy of the confinement. The confinement is modeled to be isotropic as suggested by the experiments. The second term is the mutual electrostatic energy due to the (shielded) Yukawa interaction. The shielding strength $\kappa=b / \lambda_{\mathrm{D}}$ is usually given as the ratio of interparticle distance $b$ and shielding length $\lambda_{\mathrm{D}}$.

Figure 4 shows the occupation number $N_{\mathrm{s}}$ of shell number $s$ for the ground state configuration of a Yukawa ball with $N$ particles. The occupation number is shown here as a function of $N^{2 / 3}$ (which scales as the surface area) for different 


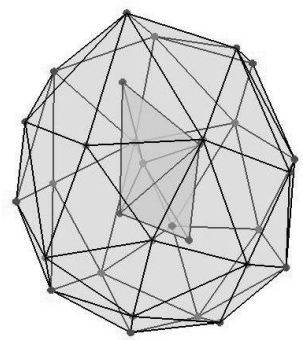

$\mathrm{N}=31$

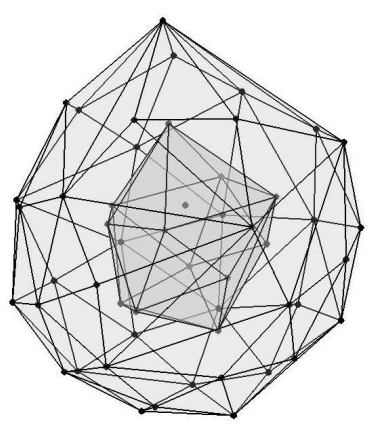

$\mathrm{N}=52$

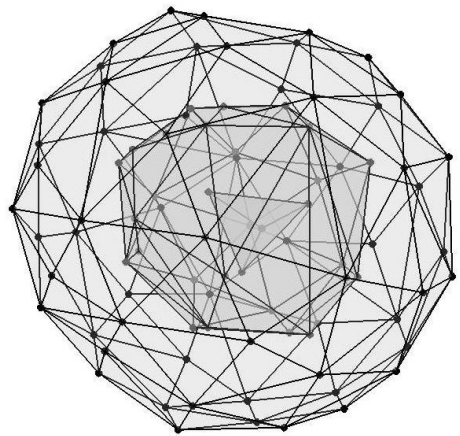

$\mathrm{N}=91$

FIGURE 3. Bond model of the structure of 3 observed Yukawa balls with $N=31,52$ and 91 . Note the concentric shell structure

screening strengths. The two cases of pure Coulomb $(\kappa=0)$ and Yukawa interaction with $\kappa=0.64$ are presented, here.

First, systematic deviations between pure Coulomb and Yukawa interaction are seen for large $N$. For Yukawa interaction, the occupation number of the outer shell is reduced compared to pure Coulomb interaction whereas the inner shells show a denser population. The reason for this is that, for pure Coulomb interaction, the force on a charge inside a homogeneously charged sphere vanishes. For the short-range Yukawa interaction this does not hold any more. Instead a force towards the center remains. This means, that for inner shells the inward remaining force from outer shells has to be balanced by a higher charge density (i.e. occupation number) on the inner shells [49, 50]. The experimental data points of the occupation numbers clearly support a Yukawa interaction with $\kappa=0.64$. It should be noted that small clusters with $N<100$ have been observed with the stereoscopic system described above. Larger systems have been recorded by the scanning technique.

As can be seen from Fig. 4 the deviation between pure Coulomb and Yukawa interaction is most obvious for large particle numbers. For small particle numbers no such deviation is seen for the range of screening strengths of interest, here. However, there is no obvious reason why the principle, that short range interaction leads to overpopulation of inner shells, should not apply in small systems.

\section{METASTABLE CONFIGURATIONS}

This ambiguity can be resolved by investigating the configurations of ground and metastable states of small clusters. It is well known that for many particle numbers metastable configurations exist with energies moderately higher than the ground state [46]. To access these different metastable states in our experiments, we have repeatedly produced clusters of fixed particle number.

To produce these different metastable configurations, the following procedure was applied. After having trapped a cluster, a well adjusted variation of rf power allows to interrupt the particle confinement for a few seconds which destroys the Yukawa ball. However, before the particles leave the discharge, we reestablished the trapping regime and the particles form a new stable cluster. This experimental artifice enables us to fill the trap several times with the same particles at the same plasma properties, but without any memory of the previous configuration.

The results of the repeated trapping experiment for a $N=31$ cluster are shown in Fig. 5 . A number of 37 realizations of the cluster have been generated at exactly the same plasma and confinement conditions. All clusters are found to consist of two shells, see Fig. 3 for comparison. However, the shell population differs among these clusters. Clusters with $N_{\mathrm{i}}=4,5$ and 6 particles on the inner shell are observed. The particle arrangement on the inner shell is given in Fig. 5. The observed structures are in perfect agreement with those expected from geometric considerations, namely a tetrahedron for $N_{\mathrm{i}}=4$, a double tetrahedron for $N_{\mathrm{i}}=5$, and a bipyramid for $N_{\mathrm{i}}=6$. As shown by the Voronoi cells in Fig. 5, the particles on the outer shell arrange in an organized pattern of hexagons and pentagons as required for a hexagonal lattice bent onto a sphere. Hence, well defined crystalline clusters of different (metastable) states $(4,27)$, $(5,26)$ and $(6,25)$ have been reliably produced in the experiment. 


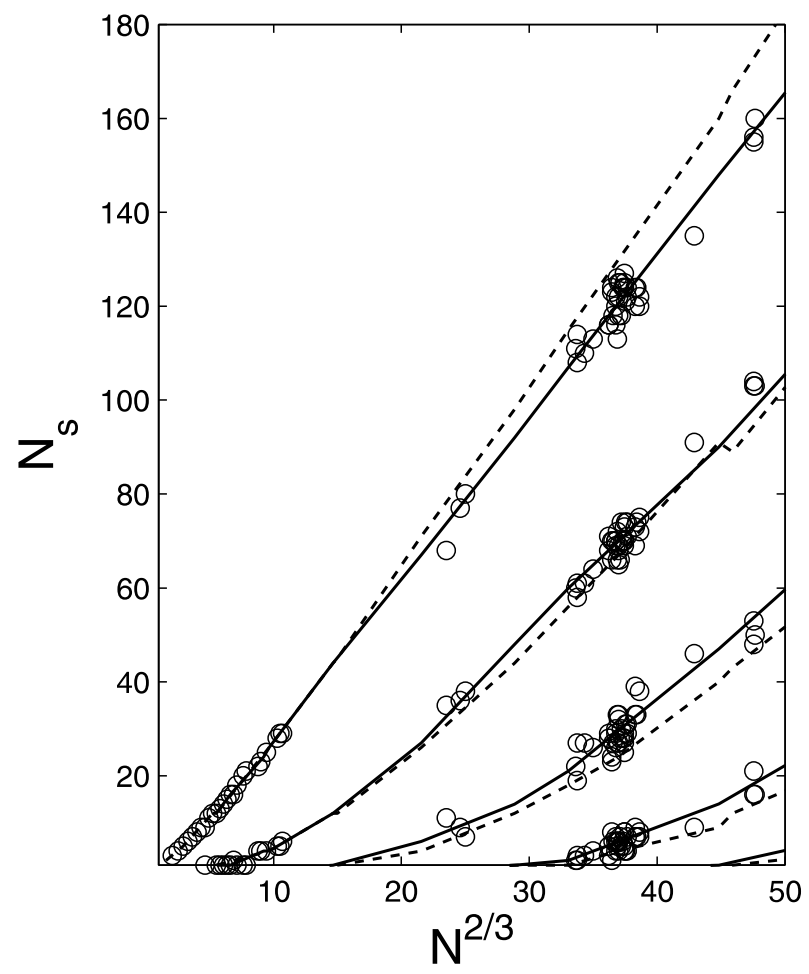

FIGURE 4. Occupation number $N_{\mathrm{S}}$ of shell number $s$ as a function of $N^{2 / 3}$. The dashed line indicates pure Coulomb interaction $(\kappa=0)$, the solid line is for shielded Yukawa interaction with $\kappa=0.64$. The symbols denote occupation numbers from experimental Yukawa balls. The data for $N<100\left(N^{2 / 3}<20\right)$ are obtained from stereoscopic techniques [43, 44], for $N>100$ from nonsimultaneous scanning techniques [36].

Interestingly, the different configurations have decisively different probability of realization. The configuration $(5,26)$ occurred in 23 out of 37 cases $(62 \%)$, the configuration $(4,27)$ in 13 cases, and the configuration $(6,25)$ was seen only once. Note that the metastable configuration $(5,26)$ appears much more frequently than the ground state $(4,27)$. It should be noted, that the configuration $(4,27)$ is the ground state for $\kappa<1.5$.

To clarify the experimental observations, we have performed MD simulations of the $N=31$ cluster. For the simulation, the parameters obtained from previous investigations have been used [51]: The dust charge is $Z=2000$ as required from the the crystalline state of the cluster. This is in agreement with previous investigations [48, 42]. The resonance frequency of the $3 \mathrm{D}$ isotropic parabolic trapping potential is found from previous experiments [42] to be $\omega_{0}=39 \mathrm{~s}^{-1}$. The cluster is taken to be at room temperature $T=300 \mathrm{~K}$.

From a variation of the screening length $\lambda_{\mathrm{D}}$ in the simulation, we can study the influence of short-range forces on the probability to form certain configurations. A number of 200 runs for each setting of the screening length has been used to derive the probability of appearance of the different configurations. The simulation is started from random particle positions to mimic the experimental loss and recovery of the confinement.

The probability to find a certain metastable configuration in the simulation is shown in Fig. 6. The configuration $(3,28)$, which has not been observed in the experiment, is found to have a non-vanishing probability only for $\lambda_{\mathrm{D}}>1000 \mu \mathrm{m}$. In contrast, the experimentally observed configuration $(6,25)$ only appears for $\lambda_{\mathrm{D}}<600 \mu \mathrm{m}$. Near $\lambda_{\mathrm{D}}=400 \mu \mathrm{m}(\kappa=1.1)$ the simulated probabilities are in a range close to the observed probability distribution. This value of the screening length is also derived from plasma simulations under similar conditions [42].

It is interesting to note that, as in the experiment, in the simulation the metastable state $(5,26)$ is observed substantially more frequently than the ground state $(4,27)$. The energy of the metastable configurations $(5,26)$ and $(6,25)$ is about $24 \mathrm{meV}$ and $49 \mathrm{meV}$ higher than the ground state, respectively. Thus, it is reasonable for dust clusters at room temperature that the metastable states are populated to a substantial degree.

Concluding, the above findings mean that with reduced screening length an increased probability exists to find clusters with higher population on the inner shell. Thus, on average a higher density on the inner shells is also found 
$(4,27)$

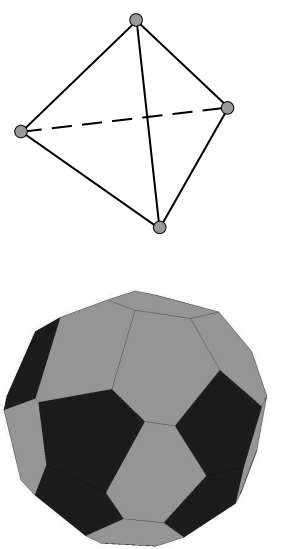

$(5,26)$

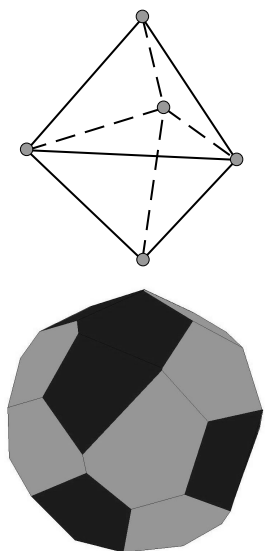

$(6,25)$
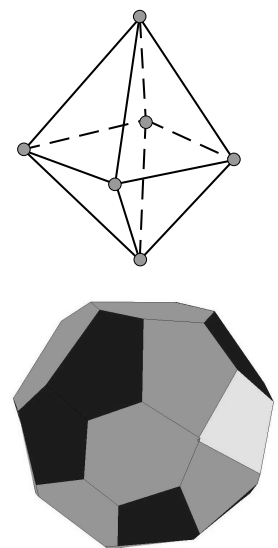

FIGURE 5. Structure of metastable configurations of the $N=31$ cluster. In the upper row, average structure of the inner shell is shown. The particle arrangement is given for the observed clusters with configurations $(4,27)$, $(5,26)$ and $(6,25)$. In the lower row the Voronoi analysis (dark gray: pentagons, medium gray: hexagons, light gray: defect) of the corresponding outer shell is presented. All plots show experimental results.

for small clusters, which is in line with the results for the larger clusters.

\section{SUMMARY}

Dusty plasmas are very well suited for the investigation of crystallized extended and finite dust arrangements. The dynamical processes appear on a time scale slow enough to be recorded by video microscopy. The dust systems can be studied on the kinetic level of individual particles.

Here, special attention has been paid to the formation of 3D finite spherical systems. With our stereoscopic imaging setup clusters with up to 100 particles have been analyzed.

These Yukawa balls show a concentric shell structure. For large clusters, the occupation numbers of the different cluster shells show a clear overpopulation of inner shells on the expense of outer shells compared to the case of pure Coulomb interaction. This is a direct consequence of the short-range Yukawa interaction. For smaller clusters, generally the ground state configuration for Coulomb and shielded Yukawa interaction agree for the range of screening strengths typically found in dusty plasmas.

However, our simulations show that the probability to observe a certain (metastable) configuration strongly depends on the interaction range: configurations with larger particle number on the inner shell are preferred for smaller shielding lengths $\lambda_{\mathrm{D}}$. This is clearly seen in the experiment for the specific case of a $N=31$ Yukawa ball where the metastable configuration $(5,26)$ is found much more frequently than the ground state $(4,27)$. Thus, the observed distribution of the configurations clearly implies a screened particle interaction with, on average, higher population of the inner shells and lower population on the outer. Hence, the dominance of short-range interaction has a significant influence on the structure even for few-particle systems. Essentially, our results show that the probability of various configurations of small clusters is directly related to the interparticle interaction.

Besides their key role in understanding the structural properties of small clusters they are of enormous importance in melting processes, because transitions among metastable states mark the onset of melting. Since smaller systems melt at lower temperatures than larger systems [52] small Yukawa balls promise to serve as ideal systems to gain insight into the microscopic processes of phase transitions in finite strongly coupled systems. 


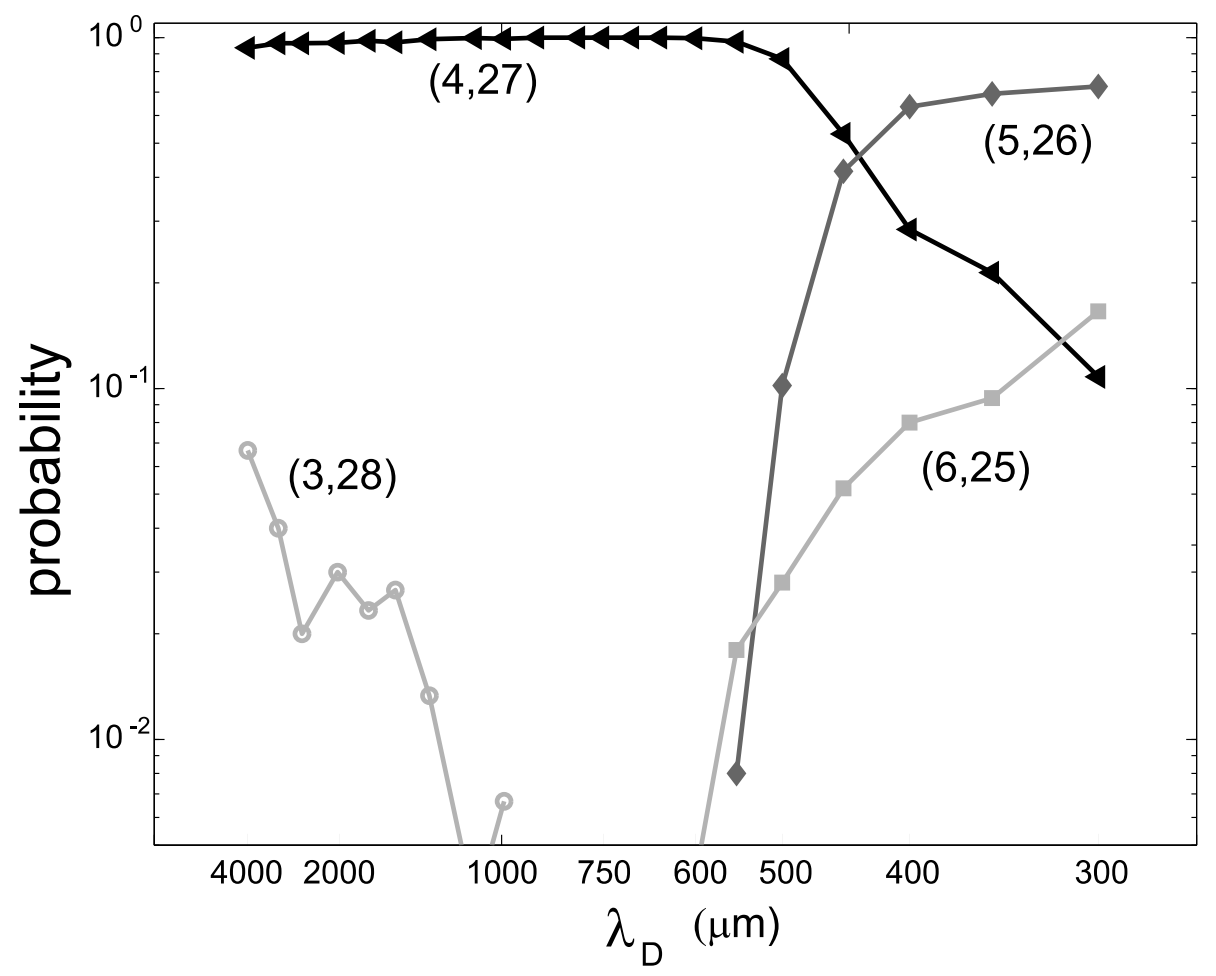

FIGURE 6. Probability of realization of configurations with $N_{\mathrm{i}}=3,4,5$ and 6 particles on the inner shell for a $N=31$ cluster as a function of screening length $\lambda_{\mathrm{D}}$.

\section{ACKNOWLEDGMENTS}

Financial support by the Deutsche Forschungsgemeinschaft via SFB-TR24 grants A2, A3, A5 and A7 is gratefully acknowledged. We thank H. Baumgartner for helpful discussion.

\section{REFERENCES}

1. F. Verheest, Waves in Dusty Space Plasmas, Kluver Academic Publishers, Dordrecht, 2000.

2. A. Bouchoule, editor, Dusty Plasmas, John Wiley \& Sons, Chichester, 1999.

3. P. K. Shukla, and A. A. Mamun, Introduction to Dusty Plasma Physics, Institute of Physics Publishing, Bristol, 2002.

4. A. Piel, and A. Melzer, Plasma Phys. Control. Fusion 44, R1-R26 (2002).

5. J. H. Chu, J.-B. Du, and L. I, J. Phys. D: Appl. Phys. 27, 296-300 (1994).

6. H. Thomas, G. E. Morfill, V. Demmel, J. Goree, B. Feuerbacher, and D. Möhlmann, Phys. Rev. Lett. 73, $652-655$ (1994).

7. Y. Hayashi, and K. Tachibana, Jpn. J. Appl. Phys. 33, L804 (1994).

8. J. H. Chu, and L. I, Phys. Rev. Lett. 72, 4009-4012 (1994).

9. A. Melzer, T. Trottenberg, and A. Piel, Phys. Lett. A 191, 301-308 (1994).

10. T. Trottenberg, A. Melzer, and A. Piel, Plasma Sources Sci. Technol. 4, 450-458 (1995).

11. J. Pieper, J. Goree, and R. Quinn, Phys. Rev. E 54, 5636 (1996).

12. M. Zuzic, A. V. Ivlev, J. Goree, G. E. Morfill, H. M. Thomas, H. Rothermel, U. Konopka, R. Sütterlin, and D. D. Goldbeck, Phys. Rev. Lett. 85, 4064 (2000).

13. A. Melzer, V. A. Schweigert, I. V. Schweigert, A. Homann, S. Peters, and A. Piel, Phys. Rev. E 54, 46-49 (1996).

14. A. Melzer, A. Homann, and A. Piel, Phys. Rev. E 53, 2757 (1996).

15. H. Thomas, and G. E. Morfill, Nature 379, 806-809 (1996).

16. G. E. Morfill, A. Ivlev, and J. Jokipii, Phys. Rev. Lett. 83, 971 (1999).

17. M. Klindworth, A. Piel, and A. Melzer, Phys. Rev. Lett. 93, 195002 (2004).

18. A. Piel, M. Klindworth, O. Arp, A. Melzer, and M. Wolter, Phys. Rev. Lett. 97, 205009 (2006).

19. W. Suzukawa, R. Ikada, Y. Tanaka, and S. Iizuka, Appl. Phys. Lett. 88, 121503 (2006). 
20. A. Nefedov, G. Morfill, V. Fortov, H. Thomas, H. Rothermel, T. Hagl, A. Ivlev, M. Zuzic, B. Klumov, and A. L. et al., New J. Phys. 5, 33 (2003).

21. V. E. Fortov, O. S. Vaulina, O. F. Petrov, V. I. Molotkov, A. V. Chernyshev, A. M. Lipaev, G. Morfill, H. Thomas, H. Rothermel, S. A. Khrapak, Y. P. Semenov, A. I. Ivanov, S. K. Krikalev, and Y. P. Gidzenko, JETP 96, 704 (2003).

22. V. V. Yaroshenko, B. M. Annaratone, S. A. Khrapak, H. M. Thomas, G. E. Morfill, V. E. Fortov, A. M. Lipaev, V. I. Molotkov, O. Petrov, A. I. Ivanov, and M. V. Turin, Phys. Rev. E 69, 066401 (2004).

23. M. Mikikian, and L. Boufendi, Phys. Plasmas 11, 3733 (2004).

24. M. Kretschmer, S. A. Khrapak, S. K. Zhdanov, H. M. Thomas, G. E. Morfill, V. E. Fortov, A. M. Lipaev, V. I. Molotkov, A. I. Ivanov, and M. V. Turin, Phys. Rev. E 71, 056401 (2005).

25. J. Goree, G. Morfill, V. Tsytovich, and S. V. Vladimirov, Phys. Rev. E 59, 7055 (1999).

26. V. Tsytovich, S. V. Vladimirov, G. Morfill, and J. Goree, Phys. Rev. E 63, 056609 (2001).

27. V. Tsytovich, Physica Scripta T89, 89-94 (2001).

28. S. Vladimirov, V. N. Tsytovich, and G. E. Morfill, Phys. Plasmas 12, 052117 (2005).

29. M. Rubin-Zuzic, G. E. Morfill, A. V. Ivlev, R. Pompl, B. A. Klumov, W. Bunk, H. M. Thomas, H. Rothermel, O. Havnes, and A. Fouquet, Nature Phys. 2, 181 (2006).

30. A. Ivlev, G. Morfill, and U. Konopka, Phys. Rev. Lett. p. 195502 (2002).

31. A. Ivlev, M. Kretschmer, M. Zuzic, G. Morfill, H. Rothermal, H. M. Thomas, V. E. Fortov, V. I. Molotkov, A. P. Nefedov, A. M. Lipaev, O. F. Petrov, Y. M. Baturin, A. I. Ivanov, and J. Goree, Phys. Rev. Lett. 90, 055003 (2003).

32. W.-T. Juan, Z.-H. Huang, J.-W. Hsu, Y.-J. Lai, and L. I, Phys. Rev. E 58, 6947 (1998).

33. M. Klindworth, A. Melzer, A. Piel, and V. Schweigert, Phys. Rev. B 61, 8404 (2000).

34. A. Melzer, Phys. Rev. E 67, 016411 (2003).

35. T. E. Sheridan, Phys. Rev. E 72, 026405 (2005).

36. O. Arp, D. Block, A. Piel, and A. Melzer, Phys. Rev. Lett. 93, 165004 (2004).

37. A. Homann, A. Melzer, S. Peters, R. Madani, and A. Piel, Phys. Rev. E 56, 7138 (1997).

38. A. Melzer, Phys. Rev. E 73, 056404 (2006).

39. B. Liu, K. Avinash, and J. Goree, Phys. Rev. Lett. 91, 255003 (2003).

40. B. Liu, and J. Goree, Phys. Rev. E 71, 046410 (2005).

41. T. Antonova, B. M. Annaratone, D. D. Goldbeck, V. Yaroshenko, H. M. Thomas, and G. E. Morfill, AIP Conf. Proc. 799, 299 (2005).

42. O. Arp, D. Block, M. Klindworth, and A. Piel, Phys. Plasmas 12, 122102 (2005).

43. S. Käding, and A. Melzer, Phys. Plasmas 13, 090701 (2006).

44. S. Käding, Y. Ivanov, and A. Melzer, IEEE Trans. Plasma Sci. 13, 090701 (2007).

45. R. W. Hasse, and V. V. Avilov, Phys. Rev. A 44, 4506 (1991).

46. P. Ludwig, S. Kosse, and M. Bonitz, Phys. Rev. E 71, 046403 (2005).

47. H. Totsuji, T. Ogawa, C. Totsuji, and K. Tsuruta, Phys. Rev. E 72, 036406 (2005).

48. M. Bonitz, D. Block, O. Arp, V. Golubnychiy, H. Baumgartner, P. Ludwig, A. Piel, and A. Filinov, Phys. Rev. Lett. 96, 075001 (2006).

49. C. Henning, H. Baumgartner, A. Piel, P. Ludwig, V. Golubnichiy, M. Bonitz, and D. Block, Phys. Rev. E 74, 056403 (2006).

50. C. Henning, P. Ludwig, A. Filinov, A. Piel, and M. Bonitz, Phys. Rev. E 76, 036404 (2007).

51. D. Block, S. Käding, A. Melzer, A. Piel, H. Baumgartner, and M. Bonitz, submitted to Phys. Rev. Lett. (2007).

52. J. P. Schiffer, Phys. Rev. Lett. 88, 205003 (2002). 